\title{
Outcomes of non-acute coronary syndrome patients discharged from the emergency department with troponin positivity
}

\author{
Nathan W. Brunner, MD*; Frank X. Scheuermeyer, MD, MHSc ${ }^{\dagger}$; Eric Grafstein, $\mathrm{MD}^{\dagger}$; \\ Krishnan Ramanathan, $\mathrm{MB}, \mathrm{ChB}^{*}$
}

\section{ABSTRACT}

Background: Cardiac troponin elevation portends a worse prognosis in diverse patient populations. The significance of troponin elevation in patients discharged from emergency departments (EDs) without inpatient admission is not well known. Methods: Patients without a diagnosis of acute coronary syndrome discharged from two EDs between April 1, 2006, and December 31, 2007, with an abnormal cardiac troponin (troponin positive [TP]) were compared to a troponin-negative (TN) cohort matched for age, sex, and primary discharge diagnosis. Outcomes were obtained by linking with a regional ED and a provincial vital statistics database and adjusted for the following: estimated glomerular filtration rate, do-notresuscitate status, history of coronary artery disease, Canadian Triage and Acuity Scale, and left ventricular hypertrophy on electrocardiography. The primary outcome was a composite of death or admission to hospital within 1 year.

Results: Our total cohort $(n=344)$ consisted of 172 TP and 172 TN patients. In the univariate analysis, TP patients had a higher rate of the primary outcome (OR 3.2, 95\% Cl 2.1-5.0, $p$ $<0.001)$ and both of its components $(p<0.001)$. After adjusting for covariates, positive troponin remained an independent predictor of the primary outcome (OR 2.1, 95\% $\mathrm{Cl} 1.3-3.4, p=0.005$ ) and inpatient admission (OR 2.0,95\% Cl 1.2-3.4, $p=0.006)$. There was no significant difference in death (OR 1.3, 95\% $\mathrm{Cl} 0.6-2.9, p=0.5$ ) after adjustment.

Conclusions: A positive troponin assay during ED stay in discharged patients is an independent marker for risk of subsequent admission. Our findings suggest that the prognostic power of an abnormal troponin extends to patients discharged from the ED.

\section{RÉSUMÉ}

Contexte: Des taux élevés de troponine cardiaque sont de mauvais augure en ce qui concerne le pronostic dans divers groupes de patients; toutefois, on ne connaît pas bien la portée des taux élevés de troponine chez les patients non hospitalisés, sortis du service des urgences (SU).
Méthode: Des patients ne souffrant pas d'un syndrome coronarien aigu et sortis de deux SU, du 1er avril 2006 au 31 décembre 2007, mais ayant un taux anormal de troponine (TAT) cardiaque ont été comparés à des patients ayant un taux normal de troponine (TNT) et appariés selon l'âge, le sexe, et le principal diagnostic au moment du congé. Les résultats ont été obtenus par l'établissement d'un lien entre la base de données du SU régional et la base de données provinciale sur l'état civil et rajustés pour tenir compte des facteurs suivants: le taux de filtration glomérulaire estimé, I'ordonnance de non-réanimation, les antécédents de coronaropathie, l'Échelle canadienne de triage et de gravité, et la présence d'hypertrophie ventriculaire gauche à l'électrocardiographie. Le principal critère d'évaluation consistait en un résultat mixte, composé de mortalité ou d'hospitalisations au bout de 1 an.

Résultats: La cohorte totale de sujets ( $n=344$ ) comptait 172 malades ayant un TAT et 172 malades ayant un TNT. D'après I'analyse unidimensionnelle, les patients qui avaient un TAT ont obtenu un résultat plus élevé au principal critère d'évaluation (risque relatif approché [RRA]: 3.2 ; IC à 95\%: 2.1-5.0; $p<0.001$ ) ainsi qu'en ce qui concerne les deux événements composant ce critère $(p<0.001)$ que les patients qui avaient un TNT. Même après le rajustement des covariables, les taux anormaux de troponine sont restés un facteur prévisionnel indépendant du principal critère d'évaluation (RRA: 2.1; IC à 95\%: 1.3-3.4; $p=0.005$ ) et d'hospitalisation (RRA: 2.0; IC à 95\%: 1.2-3.4; $p=0.006$ ). Enfin, il n'y avait pas d'écart important au regard de la mortalité (RRA 1.3; IC à 95\%: $0.6-2.9 ; p=0.5$ ) après le rajustement.

Conclusions: Des taux élevés de troponine durant un séjour au SU chez les patients sortants sont un marqueur indépendant du risque d'hospitalisation ultérieure. Ainsi, d'après les résultats de l'étude, la puissance pronostique des taux anormaux de troponine s'applique aux patients sortis du SU.

Keywords: discharge, mortality, outcomes, troponin

From the Departments of * Medicine and HEmergency Medicine, St. Paul's Hospital and the University of British Columbia, Vancouver, BC.

Correspondence to: Dr. Krishnan Ramanathan, 1081 Burrard Street, B 475, Vancouver, BC V6Z 1Y6; mailto: kramanathan@providencehealth.bc.ca. This article has been peer reviewed. 
Troponin positivity is known to be an independent predictor of adverse outcomes in many different populations, including patients with acute coronary syndrome (ACS), ${ }^{1,2}$ congestive heart failure, ${ }^{3,4}$ arrhythmia, ${ }^{5}$ pulmonary embolism, ${ }^{6,7}$ chronic obstructive pulmonary disease, ${ }^{8}$ renal failure,, 10 sepsis, ${ }^{11}$ and intracranial hemorrhage. ${ }^{12,13}$ Postprocedure troponin positivity has been associated with worse outcomes following percutaneous coronary intervention ${ }^{14}$ and both cardiac ${ }^{15,16}$ and noncardiac surgery.

Although the association of troponin positivity and worse outcomes in patients admitted to hospital is well established, ${ }^{17-19}$ it is unclear whether this association extends to patients judged to be suitable for discharge from the emergency department (ED). Understanding how troponin positivity affects patient outcomes independently of other clinical variables is imperative when determining appropriate patient disposition and subsequent follow-up.

This study investigated differences in outcomes between troponin-positive (TP) and troponin-negative (TN) patients discharged from EDs by matching all TP patients with age-, sex-, and diagnosis-matched TN patients. We hypothesized that TP patients would have worse 1-year outcomes with respect to death and hospital admission than their TN counterparts.

\section{METHODS}

\section{Study design and setting}

This was a retrospective matched cohort study and medical record review undertaken at two universityaffiliated urban EDs. Hospital A is a large tertiary referral centre with a 24-hour cardiac catheterization laboratory and surgical capability; it has an annual ED census of 65,000 . Hospital B is a community centre with 30,000 ED visits per year. The ethics committees of Providence Health Care and the University of British Columbia approved the use of clinical data. This study did not receive external funding.

\section{Inclusion and exclusion criteria}

Both EDs share a real-time electronic database that tracks all patient visits, demographics, consultations, the results of all diagnostic investigations, and ED discharge diagnoses. All patients of at least 16 years of age discharged from either ED from April 1, 2006, to
December 31, 2007, with at least one positive troponin assay obtained during their ED visit were identified from this database and comprised the troponinpositive (TP) cohort.

This administrative database also identified patients who were admitted to hospital, transferred to another hospital, who left against medical advice, died in the $\mathrm{ED}$, or did not possess a provincial health identification number, and all such patients were excluded. In addition, all patients with a listed primary diagnosis of ACS (unstable angina, non-ST segment elevated myocardial infarction [non-STEMI], and ST segment elevated myocardial infarction) on discharge from the ED were excluded because a negative prognosis associated with troponin positivity in this population is well known. We did not exclude patients with vague diagnoses of nonspecific chest pain because these diagnoses imply that the discharging physician was not convinced of ACS. An explicit chart review was undertaken to determine if patients had a history of myocardial infarction or cardiac procedure (percutaneous coronary intervention, coronary artery bypass graft, cardiac ablation, or pacemaker or implantable cardioverter-defibrillator insertion) during the previous month; these patients were excluded due to potential spurious troponin elevation. ${ }^{15,20,21}$

At both EDs, the approach to potentially ischemic chest pain is set by protocol. Myocardial infarction is defined, in a manner consistent with the universal definition of myocardial infarction,,$^{22}$ as a troponin elevation greater than the 99th percentile of the upper reference limit with a greater than 20\% change between serial measurements, or troponin elevation with electrocardiographic (ECG) changes in keeping with myocardial infarction. During structured chart review, any subjects who were discharged with a primary diagnosis of chest pain and who met the above criteria were excluded from the subsequent analysis.

\section{ED procedures}

On presentation to the ED, the Canadian Triage and Acuity Scale (CTAS), ${ }^{23}$ a standardized, reliable, and reproducible measure of acuity on presentation to an ED, was recorded for all patients. All patients were assessed by both a credentialed specialist in emergency medicine and a registered emergency nurse and underwent ECG, laboratory, and radiologic investigations at 
the attending physician's discretion. The decision to order the troponin assay was made by the attending ED physician according to his or her clinical judgment. The decision to discharge a patient could be undertaken by either the emergency physician or a consultant, but the discharge diagnosis was assigned by the emergency physician.

During the study period, both study sites were in the process of transitioning from the Roche Elecsys 2010 Troponin T Stat assay (Roche Elecsys, Laval, QC) to the Bayer Centaur Troponin I-Ultra assay (Siemens Health Care Diagnostics, Deerfield, IL). Both assays were in clinical use during the study period. In our laboratories, a value of greater than $0.05 \mu \mathrm{g} / \mathrm{L}$ exceeds the 99th percentile for both assays used. During the study period, both sites used $0.05 \mu \mathrm{g} / \mathrm{L}$ as the threshold value for reporting either troponin assay as positive.

\section{Procedure for matching cohorts}

Matching TP patients to TN counterparts was performed as follows: for every TP patient, a list of candidate TN subjects was generated from the ED database. TN and TP patients were matched for age (within 5 years), sex, and the exact discharge diagnosis coded on the patient's electronic chart. If multiple candidate $\mathrm{TN}$ subjects were present, one was selected using a random number generator. If no appropriate matches were found, the TP subject's chart was independently reviewed by two physicians (N.W.B., F.X.S.) who were blinded to all outcomes, and the discharge diagnosis was revised to reflect a broader diagnosis to facilitate matching. Any disagreements between the two physicians were adjudicated by a third physician (E.G.).

\section{Medical record review}

Chart reviews for $\mathrm{TP}$ and $\mathrm{TN}$ subjects were carried out in a structured manner as described by Gilbert and colleagues, ${ }^{24}$ although reviewers were not blinded to the study hypothesis. Two trained physician reviewers (one internal medicine specialist/cardiology fellow and one emergency physician) independently abstracted chart data and entered them into a specially designed Microsoft Excel 2007 (Microsoft Corp., Redmond, WA) spreadsheet.

The admission history and all available historical patient records (dating to 1999, the commencement of electronic charting at our institutions) were reviewed to determine if the patients had a history of myocardial infarction or cardiac procedure within the last 30 days to exclude these patients from analysis.

Other information included CTAS score and comorbidities including coronary artery disease (CAD), report of left ventricular hypertrophy (LVH) on ECG, and decreased ejection fraction $(<45 \%)$ or aortic stenosis (valve area $<1.5 \mathrm{~cm}^{2}$ ). Each patient's lowest estimated glomerular filtration rate (eGFR) and the highest measured troponin value on their index ED visit were recorded. Charts were scrutinized to determine whether a do not resuscitate (DNR) order (code status) was present at the time of the index visit. Measures of interobserver reliability were generated by comparing the values obtained for LVH on ECG and a history of CAD (as dichotomous yes/no outcomes) by the two independent reviewers for $10 \%$ of the charts chosen at random.

Patients missing data on the eGFR or LVH on ECG were excluded from the adjusted analysis. If no information was found regarding the presence or absence of a history of CAD, the patient was assumed to have no history of CAD. If there was no comment regarding DNR status, the patient was assumed to wish a maximum level of therapeutic intervention. Cases with discrepant data were referred to an independent blinded adjudicator.

\section{Follow-up and outcomes}

Using each patient's unique identifier (the provincial health care number), patients were linked with both the regional $\mathrm{ED}$ database, which tracks all regional ED visits and hospital admissions, and the provincial vital statistics database to ascertain mortality. Our primary outcome was a composite of all-cause mortality or inpatient admission within 1 year of the index visit. Secondary outcomes included the components of the primary outcome and repeat ED visit within 1 month of the index visit. Only overnight admissions to hospital were included. Short-stay admissions of less than 1 day, such as those for coronary angiography or cardiac ablation, were not included.

\section{Statistical analysis}

SPSS software version 19 (SPSS Inc., Chicago, IL) was used for all statistical analysis. Baseline characteristics 
are reported as means and standard deviations for continuous variables and percentages for categorical variables. The Student's t test was used to compare continuous variables, and the chi-square test was used to compare categorical variables. Unadjusted cumulative incidences of events were analyzed using KaplanMeier methods and compared using the log-rank test. All patients were censored at the time of an event or at 1 year following their index ED visit for the combined outcome and the end points of mortality and hospital admission, whichever came first. For the ED revisit end point, patients were censored at the time of an event or at 1 month following their index ED visit, whichever came first.

Binary logistic regression was used to calculate odds ratios and $95 \%$ confidence intervals for each outcome. We adjusted for eGFR and LVH because renal failure and $\mathrm{LVH}$ have been associated with higher rates of troponin elevation and are also associated with adverse outcomes. ${ }^{25-28} \mathrm{We}$ adjusted for $\mathrm{CAD}$, code status, and CTAS score because they are associated with adverse outcomes and factor into the ED physician's decision to discharge..$^{29,30}$ The Hosmer-Lemeshow test was used to assess goodness of fit. All reported $p$ values are two-sided, and any $p$ value $<0.05$ was deemed to be significant.

\section{RESULTS}

\section{Patient characteristics}

Our initial interrogation of the ED database yielded 222 patients discharged from the ED after having a positive troponin assay. Fifty patients were excluded for having a discharge diagnosis of ACS, leaving against medical advice, being transferred to other institutions for admission, having had a recent myocardial infarction or cardiac procedure, or having chest pain and fitting the ED definition for nonSTEMI on chart review (Figure 1). The remaining 172 patients comprised the TP cohort. The troponin I assay was used in $52 \%$ of the TP cohort, and the troponin $\mathrm{T}$ assay was used in the remainder.

Table 1 shows the most common ED discharge diagnoses for the TP cohort; they included both cardiac and noncardiac pathologies. During the study period, 6,410 patients had negative troponin assays in the ED and were discharged; the TN cohort was chosen from this group. No TP patients were unmatched from an age or sex perspective, but $28(16 \%)$ of the TP subjects had no appropriate match for the discharge diagnosis, and in these patients, the diagnosis had to be reviewed and broadened to find an appropriate match.

For the chart review, interrater reliability was good for the key variables of history of CAD $(\kappa=0.89)$ and LVH on ECG $(\kappa=0.77)$ in a random $10 \%$ sample. Although information relating to triage acuity, troponin elevation, CAD, renal failure, and $\mathrm{LVH}$ was consistently present, only $50 \%$ of patients had an echocardiogram report available on the electronic medical record system. Baseline characteristics for each cohort are shown in Table 2. TP patients were more likely to have a history of CAD, lower GFRs, and a DNR order. The median peak measured troponin was $0.14 \mu \mathrm{g} / \mathrm{L}$. Figure 2 demonstrates the distribution of the troponin values

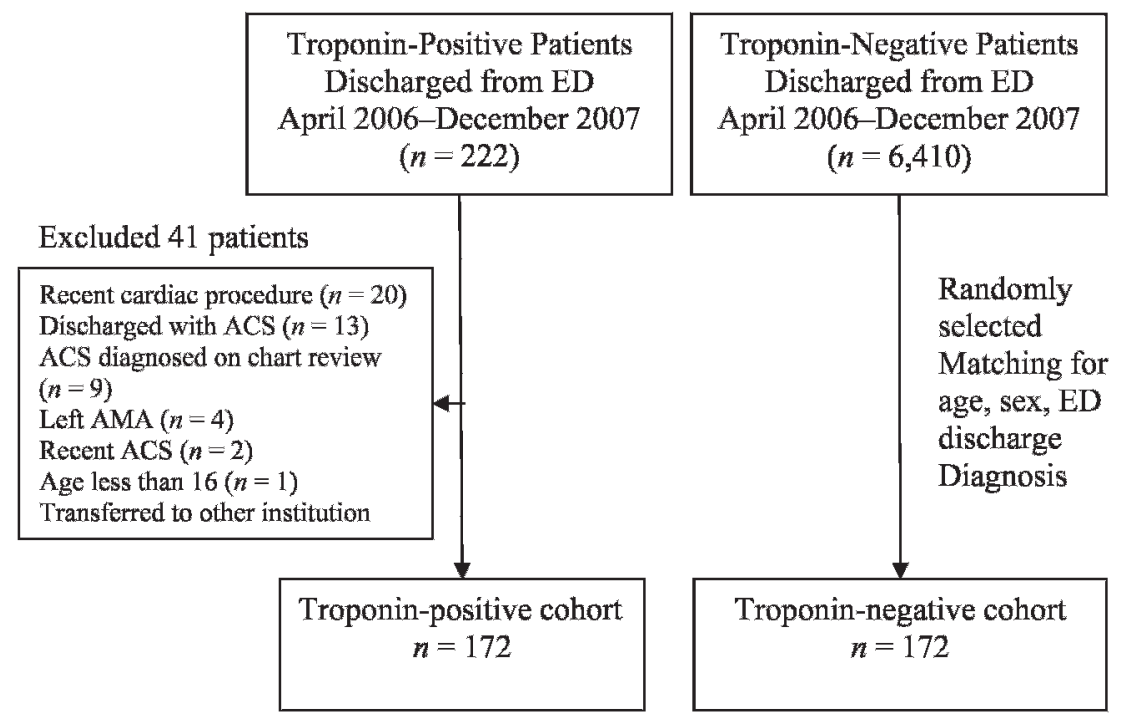

Figure 1. Study flow diagram. ACS = acute coronary syndrome; AMA = against medical advice; ED = emergency department. 


\begin{tabular}{lc|}
\hline $\begin{array}{l}\text { Table 1. Most common emergency department discharge } \\
\text { diagnoses for the troponin-positive cohort }(\boldsymbol{n}=\mathbf{1 7 2})\end{array}$ \\
\hline Diagnosis & Number of patients (\%) \\
\hline Nonspecific chest pain & $31(18)$ \\
Congestive heart failure/ & $14(8)$ \\
$\quad$ pulmonary edema & $14(8)$ \\
Infectious & $12(7)$ \\
Atrial fibrillation/flutter & $12(7)$ \\
Other arrhythmia/palpitations & $10(6)$ \\
Chronic renal failure & $10(6)$ \\
Syncope NYD & $9(5)$ \\
Asthma/chronic obstructive & \\
pulmonary disease & $8(5)$ \\
General weakness NYD & $6(3)$ \\
Abdominal pain NYD & $5(3)$ \\
Stroke/transient ischemic & \\
attack/seizure & $5(3)$ \\
Intoxication/withdrawal & $4(2)$ \\
Confusion/decreased level of & \\
consciousness & $4(2)$ \\
Musculoskeletal pain & $4(2)$ \\
Other respiratory & $3(2)$ \\
Other cardiac & $21(12)$ \\
Miscellaneous & \\
\hline NYD $=$ not yet diagnosed. &
\end{tabular}

for TP patients. As expected, there is clear clustering of the troponin measurements around the $0.05 \mu \mathrm{g} / \mathrm{L}$ threshold used by both laboratories.

\section{Unadjusted Outcomes}

In Table 3, we show the unadjusted event rates for the $\mathrm{TP}$ and TN cohorts. TP patients had substantially higher 1-year combined outcomes of death and inpatient admission- $53 \%$ versus $27 \%$ in the $\mathrm{TN}$ cohort $(p<0.001)$. Both components of the primary outcome were associated with troponin positivity, as shown in Table 3. There was a nonsignificant increase in repeat ED visits at 1 month. The Kaplan-Meier curves for the primary outcome and each of the secondary outcomes for the TP and TN cohorts are shown in Figure 3. As expected, the primary outcome was associated with CAD, eGFR, DNR status, and LVH on ECG (Appendix 1). Death at 1 year was associated with CAD, eGFR, and DNR status, and inpatient admission at 1 year was associated with CAD, eGFR, and LVH. No associations were found between CTAS score and any of the outcomes of interest.

\section{Adjusted Outcomes}

Given that the CTAS score was not significantly associated with any outcomes in the unadjusted analysis, we omitted it from our subsequent multivariate analysis. Twelve patients were missing data on covariates $(2$ in the $\mathrm{TP}$ cohort and 10 in the $\mathrm{TN}$ cohort) and were excluded. After adjusting for history of CAD, DNR status, eGFR, and LVH on ECG, the primary outcome was more common in the TP versus the TN cohort (OR 2.06, 95\% CI 1.25-3.39, $p=$ $0.005)$, as shown in Table 4. Similarly, the rate of inpatient admission at 1 year was higher for the TP versus the TN cohort after adjusting for covariates (OR 2.02, 95\% CI 1.22-3.36, $p=0.006$ ). However, there was no significant difference in death (OR 1.34, $95 \%$ CI $0.62-2.89, p=0.5$ ) or 1 -month ED revisit

\begin{tabular}{|c|c|c|c|}
\hline Characteristic & Troponin-positive & Troponin-negative & $p$ value \\
\hline Age (mean $\pm \mathrm{SD})$ & $72 \pm 16$ & $71 \pm 16$ & $0.7^{*}$ \\
\hline Female sex (\%) & 38 & 38 & $1.0^{*}$ \\
\hline CTAS score (\%) & & & 0.1 \\
\hline $1-2$ & 36 & 26 & \\
\hline 3 & 51 & 58 & \\
\hline $4-5$ & 13 & 17 & \\
\hline History of CAD & $43 \%$ & $21 \%$ & $<0.001$ \\
\hline Estimated GFR (mean \pm SD) & $52 \pm 34$ & $71 \pm 28$ & $<0.001$ \\
\hline Median troponin $\mu \mathrm{g} / \mathrm{L}$ & 0.14 & - & $<0.001$ \\
\hline Do-not-resuscitate status (\%) & 15 & 6 & 0.005 \\
\hline LVH on ECG (\%) & 19 & 13 & 0.2 \\
\hline Cardiology consultation (\%) & 30 & 5 & $<0.001$ \\
\hline
\end{tabular}




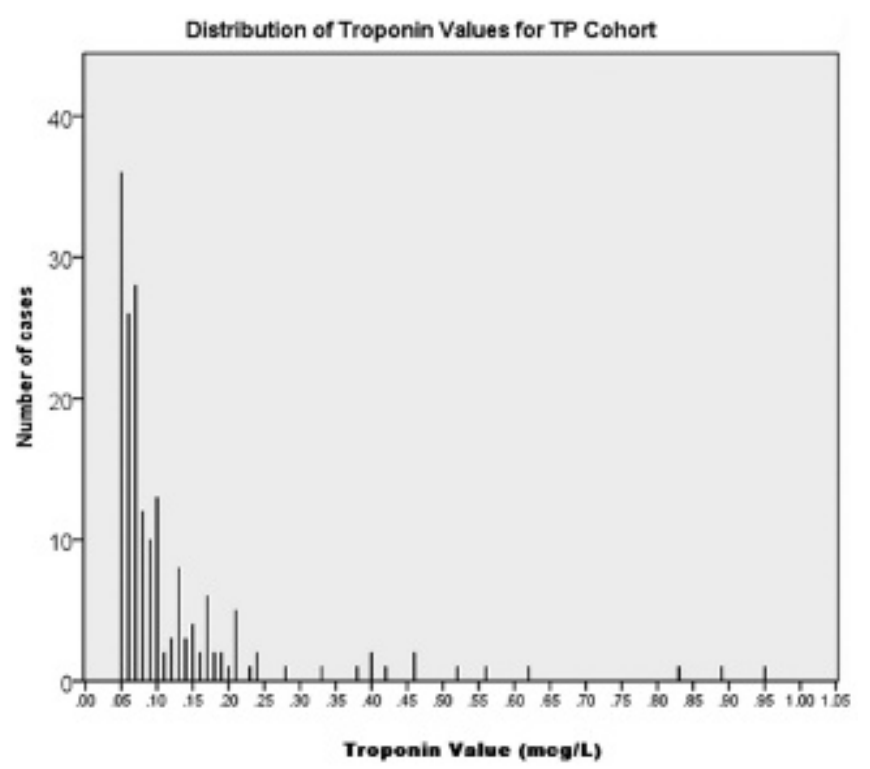

Figure 2. Distribution of troponin values for the troponinpositive cohort.

(OR 1.44, 95\% CI 0.85-2.44, $p=0.2$ ) for TP patients compared to their TN controls. Appendix 2 demonstrates the associations between outcomes and history of CAD, DNR status, eGFR, and LVH in our multivariate models. All models had adequate goodness of fit (Hosmer-Lemeshow $p>0.5$ for each).

\section{DISCUSSION}

In this matched cohort study of patients discharged from two large urban EDs, a positive troponin measurement while in the ED was independently associated with the composite of death or admission to hospital within 1 year, as well as admission to hospital within 1 year alone. To our knowledge, this represents the largest cohort study to assess the prognostic ability of troponin assays on discharge from the ED.
Among patients admitted to hospital, troponin elevation in the absence of ACS is associated with higher all-cause mortality, cardiac mortality, nonfatal myocardial infarction, and readmission for cardiac causes. ${ }^{1,5,19}$ Increases in short- and long-term mortality have been seen in critically ill patients admitted to the intensive care unit. ${ }^{31}$ Highly sensitive troponin assays were recently investigated in the ED setting and are associated with mortality in patients with suspected ischemic chest pain. ${ }^{32,33}$ A highly sensitive troponin $\mathrm{T}$ assay was also recently shown to predict mortality in a healthy outpatient population. ${ }^{34}$ Our study mirrors the above findings and suggests that troponin positivity in patients suitable for discharge from the ED also carries prognostic power.

In the absence of coronary plaque rupture, minor troponin elevation can result from mismatch between myocardial oxygen demand and oxygen supply. Detectable cardiac troponin levels may reflect a reduced homeostatic reserve to situations that increase oxygen demand (e.g., tachycardia, sepsis) or decrease oxygen supply (e.g., hypoxia, anemia). ${ }^{17,35}$ Thus, an increased rate of mortality and admission could be expected due to a decreased ability to compensate for disease. Given the association between troponin elevation and subsequent ACS noted in previous studies, it may also reflect subclinical coronary atherosclerosis that may lead to plaque rupture and myocardial infarction at a later date.

The prognostic implications of positive troponin assays on discharge from the ED have been sparingly investigated. At present, outcomes in this specific population have only been investigated in an isolated cohort study. ${ }^{36}$ Fundaro and Guzzetti found that patients discharged from a single ED had a higher rate of death, hospitalization, or repeat visit to the ED at 30 days compared to age- and sex-matched controls $(37 \%$ v. $19 \%) .{ }^{36}$ However, the study was not large

\begin{tabular}{|c|c|c|c|c|}
\hline Outcome & $\mathrm{TP}, n(\%)(n=172)$ & $\mathrm{TN}, n(\%)(n=172)$ & Odds ratio $(95 \% \mathrm{Cl})$ & $p$ value \\
\hline $\begin{array}{l}\text { Primary outcome of death and } \\
\text { hospitalization at } 1 \text { year }\end{array}$ & $92(53)$ & $46(27)$ & $3.15(2.01-4.95)$ & $<0.001$ \\
\hline Death at 1 year & $29(17)$ & $13(8)$ & $2.48(1.24-4.96)$ & 0.01 \\
\hline Hospitalization at 1 year & $87(51)$ & $43(25)$ & 3.07 (1.95-4.85) & $<0.001$ \\
\hline Repeat ED visit at 1 month & $53(31)$ & $38(22)$ & $1.63(0.98-2.57)$ & 0.06 \\
\hline
\end{tabular}


A

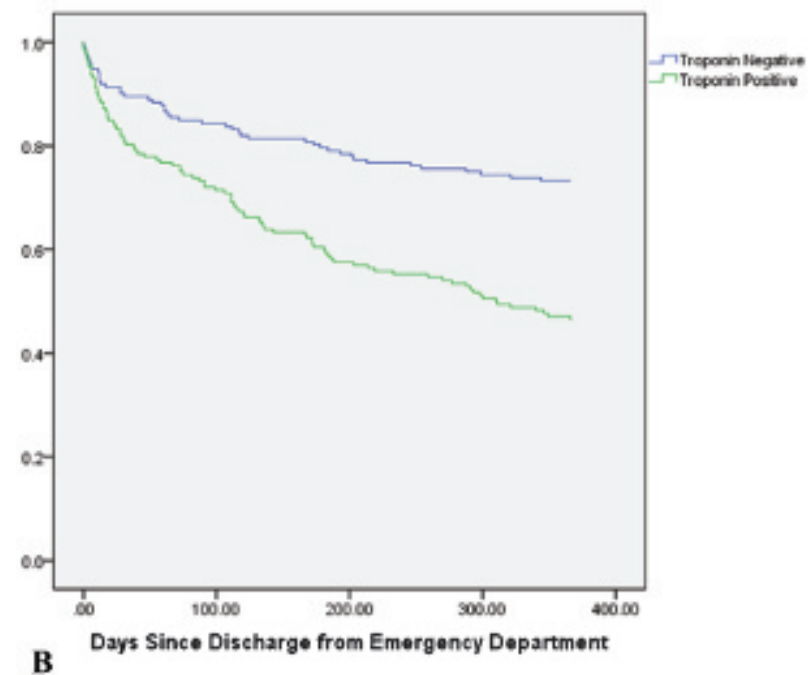

B

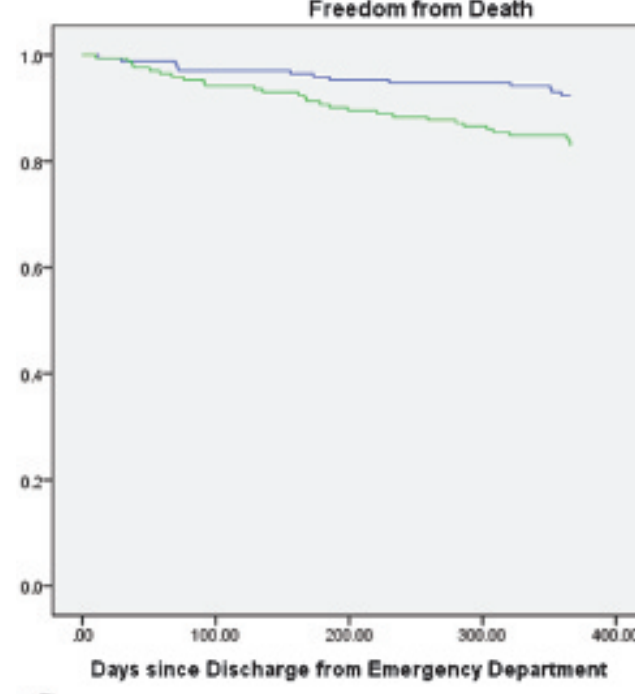

C

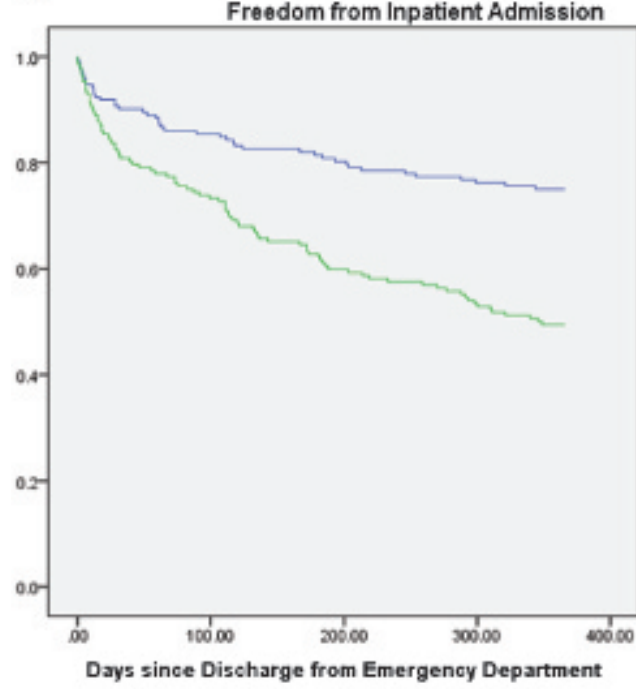

Figure 3. Kaplan-Meier curves for primary and secondary outcomes. A, Freedom from death or hospital admission. $B$, Freedom from death. $C$, Freedom from inpatient admission.

enough to permit a multivariate analysis. Multivariate analyses adjusting for baseline differences between cohorts are essential in observational studies, and our work contributes this level of evidence to the existing literature.

We demonstrated that ED troponin elevation in discharged patients is associated with subsequent hospital admission independent of age, sex, serum creatinine concentration, established history of heart disease, DNR status, CTAS score, and ECG-proven LVH. Although this list of prognosticators is by no means exhaustive, complete information on all prognostically important variables is rarely present on discharge from the ED. By matching our cohorts for age, sex, and discharge diagnosis and controlling for confounders readily available in the ED at the time of discharge, we suspect that the difference in unmeasured covariates between the two cohorts was minimized.

This study has important implications for the management of patients discharged from the ED. We have demonstrated that a minimally positive troponin assay is associated with a worse prognosis, independent of other markers of risk that are readily available on discharge from the ED. Thus, slightly positive troponin assays cannot simply be dismissed as inconsequential findings. Although there was some clustering of events immediately postdischarge, there was continued gradual separation of the Kaplan-Meier curves through the duration of follow-up. Thus, our findings indicate that a minimally elevated troponin on ED discharge may serve as a marker of general risk rather than a signal of impending myocardial infarction. If discharging a patient with a minimally positive troponin, physicians should ensure appropriate followup given the increased risk of death or admission at 1 year.

\section{LIMITATIONS}

Some limitations may affect our findings. The two cohorts (TN and TP) were dissimilar at baseline, although we took care to match for age, gender, and discharge diagnosis. We adjusted for baseline differences to mitigate these differences. Additionally, the 


\begin{tabular}{|c|c|c|c|}
\hline & OR & $95 \% \mathrm{Cl}$ & $p$ value \\
\hline Composite outcome & 2.06 & $1.25-3.39$ & 0.005 \\
\hline Death at 1 year & 1.34 & $0.62-2.89$ & 0.5 \\
\hline Inpatient admission at 1 year & 2.02 & $1.22-3.36$ & 0.006 \\
\hline Repeat ED visit at 1 month & 1.44 & $0.85-2.44$ & 0.2 \\
\hline
\end{tabular}

retrospective nature of our study may be prone to ascertainment bias in that subjects may have had different degrees of workup at the discretion of the treating physician. This variation in practice was minimized by the existence of standardized protocols, but compliance with the existing protocols was not measured.

Another significant limitation is our treatment of both troponin assays in use during the study time period as equivalent. Although both assays have been well validated for clinical use, minor variations in the assays do exist. ${ }^{35,37,38}$ The published 99th percentile limit for the Centaur Troponin I assay is $0.04 \mu \mathrm{g} / \mathrm{L}$, whereas the corresponding value for the Elecsys Troponin T assay is $0.01 \mu \mathrm{g} / \mathrm{L} .^{39,40}$ During the course of this study, both EDs used $0.05 \mu \mathrm{g} / \mathrm{L}$ as the threshold for identifying a positive test. As the goal of this study was to investigate outcomes in patients who were knowingly discharged with positive troponins, this mandated that we also use a threshold of $0.05 \mu \mathrm{g} / \mathrm{L}$ to identify TP patients in both troponin assays.

A significant number of TP patients were discharged with a diagnosis of nonspecific chest pain. This diagnosis is not ideal but is reflective of real-world clinical practice, where an ultimate diagnosis cannot always be reached in the ED. Although it is natural to question whether some of these may have been "missed ACS" presentations, the low level of troponin elevation seen makes it very unlikely that these presentations were due to rupture of an atherosclerotic plaque causing ACS.

The data on readmission or repeat visit to ED were taken from a regional database, and any of these events occurring in patients who migrated outside the health region would not have been recognized. However, this effect should be evenly distributed across both cohorts.
Our study focused on all-cause mortality and hospital admission at 1 year and does not differentiate between cardiac and noncardiac etiologies.

\section{CONCLUSIONS}

In patients discharged from two urban EDs with positive troponin assays, we noted an increased incidence of death or hospital admission at 1 year compared to controls matched for age, sex, and ED discharge diagnosis. Future study should address the causes of death and hospitalization at 1 year in this population so that this information can guide preventive interventions.

Competing interests: None declared.

\section{REFERENCES}

1. Setiadi BM, Lei H, Chang J. Troponin not just a simple cardiac marker: prognostic significance of cardiac troponin. Chin Med 7 2009;122:351-8.

2. Ottani F, Galvani M, Nicolini FA, et al. Elevated cardiac troponin levels predict the risk of adverse outcome in patients with acute coronary syndromes. Am Heart 7 2000; 140:917-27, doi:10.1067/mhj.2000.111107.

3. Setsuta K, Seino Y, Kitahara Y, et al. Elevated levels of both cardiomyocyte membrane and myofibril damage markers predict adverse outcomes in patients with chronic heart failure. Circ 7 2008;72:569-74, doi:10.1253/circj.72. $\underline{569}$.

4. Demir M, Kanadasi M, Akpinar O, et al. Cardiac troponin T as a prognostic marker in patients with heart failure: a 3-year outcome study. Angiology 2007;58:603-9, doi:10.1177/ 0003319707307344.

5. Chow GV, Hirsch GA, Spragg DD, et al. Prognostic significance of cardiac troponin I levels in hospitalized patients presenting with supraventricular tachycardia. Medicine 2010; 89:141-8, doi:10.1097/MD.0b013e3181dddb3b.

6. Becattini C, Vedovati MC, Agnellli G. Prognostic value of troponins in acute pulmonary embolism: a meta-analysis. Circulation 2010;116:427-33, doi:10.1161/CIRCULATIONAHA. 106.680421.

7. Stein PD, Matta F, Janjua M, et al. Outcome in stable patients with acute pulmonary embolism who had right ventricular enlargement and/or elevated levels of troponin I. Am 7 Cardiol 2010;106:558-63, doi:10.1016/j.amjcard.2010. 03.071 .

8. Martins CS, Rodrigues MJO, Miranda VP, et al. Prognostic value of cardiac troponin I in patients with COPD acute exacerbation. Neth 7 Med 2009;67:341-9.

9. Choy JB, Armstrong PW, Ulan RA, et al. Do cardiac troponins provide prognostic insight in hemodialysis patients? Can 7 Cardiol 2003;19:907-11.

10. McGill D, Talaulikar G, Potter JM, et al. Over time, highsensitivity TnT replaces NT-proBNP as the most powerful 
predictor of death in patients with dialysis-dependent chronic renal failure. Clin Chim Acta 2010;411:936-9, doi:10.1016/j.cca.2010.03.004.

11. Kalla C, Raveh D, Algur N, et al. Incidence and significance of a positive troponin test in bacteremic patients without acute coronary syndrome Am 7 Med 2008;121:909-15, doi:10.1016/j.amjmed.2008.05.037.

12. Garrett MC, Komotar RJ, Starke RM, et al. Elevated troponin levels are predictive of mortality in surgical intracerebral hemorrhage patients. Neurocrit Care 2010;12: 199-203, doi:10.1007/s12028-009-9245-5.

13. Naidech AM, Kreiter KT, Janjua N, et al. Cardiac troponin elevation, cardiac morbidity and outcome after subarachnoid hemorrhage. Circulation 2005;112:2851-6, doi:10.1161/ CIRCULATIONAHA.105.533620.

14. Milani RV, Fitzgerald R, Milani JN, et al. The impact of micro troponin leak on long-term outcomes following elective percutaneous coronary intervention. Catheter Cardiovasc Interv 2009;74:819-22, doi:10.1002/ ccd.22160.

15. Croal BL, Hillis GS, Gibson PH, et al. Relationship between postoperative cardiac troponin I levels and outcome of cardiac surgery. Circulation 2006;114:1468-75, doi:10. 1161/CIRCULATIONAHA.105.602370.

16. Oshima K, Kunimoto F, Takahashi T, et al. Postoperative cardiac troponin I (cTnI) level and its prognostic value for patients undergoing mitral valve surgery. Int Heart 7 2010; 51:166-9, doi:10.1536/ihj.51.166.

17. Pham MX, Whooley MA, Evans GT Jr, et al. Prognostic value of low-level cardiac troponin-I elevations in patients without definite acute coronary syndromes. Am Heart 7 2004;148:776-82, doi:10.1016/j.ahj.2004.03.058.

18. Carlson ER, Percy RF, Angiolillo DJ, et al. Prognostic significance of troponin $T$ elevation in patients without chest pain. Am 7 Cardiol 2008;102:668-71, doi:10.1016/j.amjcard. 2008.04.046.

19. Srivathsan K, Showalter J, Wilkens J, et al. Cardiovascular outcome in hospitalized patients with minimal troponin I elevation and normal creatine phosphokinase. Int 7 Cardiol 2004;97:221-4, doi:10.1016/j.ijcard.2003.08.018.

20. Alaiti MA, Maroo A, Edel TB. Troponin levels after cardiac electrophysiology procedures: review of the literature. Pacing Clin Electrophysiol 2009;32:800-10, doi:10.1111/j.1540-8159. 2009.02370.x.

21. Loeb HS, Liu JC. Frequency, risk factors, and effect on long-term survival of increased troponin I following uncomplicated elective percutaneous coronary intervention. Clin Cardiol 2010;33:E40-4, doi:10.1002/clc.20425.

22. Thygesen K, Alpert JS, White HD, et al. Universal definition of myocardial infarction. Circulation 2007;116: 2634-53, doi:10.1161/CIRCULATIONAHA.107.187397.

23. Beveridge R. CAEP issues. The Canadian Triage and Acuity Scale: a new and critical element in health care reform. Canadian Association of Emergency Physicians. 7 Emerg Med 1998;16:507-11, doi:10.1016/S0736-4679(98) 00031-6.

24. Gilbert EH, Lowenstein SR, Koziol-McLain J, et al. Chart reviews in emergency medicine research: where are the methods? Ann Emerg Med 1996;27:305-8, doi:10.1016/ $\underline{\text { S0196-0644(96)70264-0. }}$
25. Agarwal R, Light RP. Determinants and prognostic significance of electrocardiographic left ventricular hypertrophy criteria in chronic kidney disease. Clin 7 Am Soc Nephrol 2011; 6:528-36, doi:10.2215/CJN.07770910.

26. Chronic Kidney Disease Prognosis Consortium, Matsushita K, van der Velde $M$, et al. Association of estimated glomerular filtration rate and albuminuria with all-cause and cardiovascular mortality in general population cohorts: a collaborative meta-analysis. Lancet 2010;375:2073-81, doi:10.1016/S01406736(10)60674-5.

27. Hsieh BP, Pham MX, Froelicher VF. Prognostic value of electrocardiographic criteria for left ventricular hypertrophy. Am Heart 7 2005;150:161-7, doi:10.1016/j.ahj.2004. 08.041 .

28. Roderick PJ, Atkins RJ, Smeeth L, et al. CKD and mortality risk in older people: a community-based population study in the United Kingdom. Am 7 Kidney Dis 2009;53:950-60, doi:10.1053/j.ajkd.2008.12.036.

29. Dong SL, Bullard MJ, Meurer DP, et al. Predictive validity of a computerized emergency triage tool. Acad Emerg Med 2007;14:16-21, doi:10.1111/j.1553-2712.2007. tb00362.x.

30. Phillips RS, Wenger NS, Teno J, et al. Choices of seriously ill patients about cardiopulmonary resuscitation: correlates and outcomes. SUPPORT Investigators. Study to Understand Prognoses and Preferences for Outcomes and Risks of Treatments. Am 7 Med 1996;100:128-37, doi:10.1016/ $\underline{\mathrm{S} 0002-9343(97) 89450-8 .}$

31. Lim W, Cook DJ, Griffith LE, et al. Elevated cardiac troponin levels in critically ill patients: prevalence, incidence, and outcomes. Am 7 Crit Care 2006;15:280-8; quiz 289.

32. Mills NL, Churchhouse AM, Lee KK, et al. Implementation of a sensitive troponin I assay and risk of recurrent myocardial infarction and death in patients with suspected acute coronary syndrome. FAMA 2011;305:1210-6, doi:10. 1001/jama.2011.338.

33. Keller T, Zeller T, Peetz D, et al. Sensitive troponin I assay in early diagnosis of acute myocardial infarction. $N$ Engl 7 Med 2009;361:868-77, doi:10.1056/NEJMoa0903515.

34. de Lemos JA, Drazner MH, Omland T, et al. Association of troponin $\mathrm{T}$ detected with a highly sensitive assay and cardiac structure and mortality risk in the general population. FAMA 2010;304:2503-12, doi:10.1001/jama. 2010.1768 .

35. Thygesen K, Mair J, Katus H, et al. Recommendations for the use of cardiac troponin measurement in acute cardiac care. Eur Heart 7 2010;31:2197-204, doi:10.1093/eurheartj/ ehq251.

36. Fundaro C, Guzzetti S. Prognostic value of stable troponin $\mathrm{T}$ elevation in patients discharged from emergency department. 7 Cardiovasc Med (Hagerstown) 2010;11:276-80, doi:10.2459/JCM.0b013e328336ecc5.

37. Venge P, Lagerquist B, Diderholm E, et al. Clinical performance of three cardiac troponin assays in patients with unstable coronary artery disease (a FRISC II substudy). Am 7 Cardiol 2002;89:1035-41, doi:10.1016/S0002-9149(02) 02271-3.

38. Heeschen C, Deu A, Langenbrink L, et al. Analytical and diagnostic performance of troponin assays in patients 
suspicious for acute coronary syndromes. Clin Biochem 2000; 33:359-68, doi:10.1016/S0009-9120(00)00144-2.

39. Apple FS, Smith SW, Pearce LA, et al. Use of the Centaur TnI Ultra assay for detection of myocardial infarction and adverse events in patients presenting with symptoms suggestive of acute coronary syndrome. Clin Chem 2008;54: 723-8, doi:10.1373/clinchem.2007.097162.

40. Panteghini M, Pagani Y, Yeo KT, et al. Evaluation of imprecision for cardiac troponin assays at low-range concentrations. Clin Chem 2004;50:327-32, doi:10.1373/clinchem.2003.026815. 


\begin{tabular}{|c|c|c|c|}
\hline & OR & $95 \% \mathrm{Cl}$ & $p$ value \\
\hline \multicolumn{4}{|c|}{ Composite outcome (death or admission at 1 year) } \\
\hline Troponin & 3.15 & $2.01-4.95$ & $<0.001$ \\
\hline CTAS score & & & 0.7 \\
\hline $1-2$ & 0.85 & $0.46-1.59$ & \\
\hline 3 & 0.75 & $0.38-1.46$ & \\
\hline History of CAD & 3.01 & $1.88-4.81$ & $<0.001$ \\
\hline eGFR* & 0.99 & $0.98-0.99$ & $<0.001$ \\
\hline DNR status & 2.60 & $1.28-5.28$ & 0.008 \\
\hline LVH on ECG & 1.99 & $1.11-3.55$ & 0.02 \\
\hline \multicolumn{4}{|l|}{ Death at 1 year } \\
\hline Troponin & 2.48 & $1.24-4.96$ & 0.01 \\
\hline CTAS score & & & 0.4 \\
\hline $1-2$ & 0.82 & $0.34-1.94$ & \\
\hline 3 & 0.52 & $0.19-1.43$ & \\
\hline History of CAD & 4.22 & $2.15-8.27$ & $<0.001$ \\
\hline eGFR* & 0.99 & $0.98-1.00$ & 0.02 \\
\hline DNR status & 2.78 & $1.20-6.41$ & 0.02 \\
\hline LVH on ECG & 1.75 & $0.80-3.81$ & 0.2 \\
\hline \multicolumn{4}{|l|}{ Admission at 1 year } \\
\hline Troponin & 3.07 & $1.95-4.85$ & $<0.001$ \\
\hline CTAS score & & & 0.8 \\
\hline $1-2$ & 0.79 & $0.42-1.59$ & \\
\hline 3 & 0.81 & $0.41-1.47$ & \\
\hline History of CAD & 3.34 & $2.08-5.36$ & $<0.001$ \\
\hline eGFR* & 0.98 & $0.98-0.99$ & $<0.001$ \\
\hline DNR status & 1.98 & $0.99-3.97$ & 0.05 \\
\hline LVH on ECG & 2.05 & $1.15-3.68$ & 0.02 \\
\hline \multicolumn{4}{|l|}{ Revisit at 1 month } \\
\hline Troponin & 1.63 & $0.98-2.57$ & 0.06 \\
\hline CTAS score & & & 0.3 \\
\hline $1-2$ & 0.59 & $0.30-1.14$ & \\
\hline 3 & 0.70 & $0.34-1.43$ & \\
\hline History of CAD & 1.06 & $0.63-1.77$ & 0.8 \\
\hline eGFR* & 1.00 & $0.99-1.01$ & 0.9 \\
\hline DNR status & 1.41 & $0.72-3.18$ & 0.3 \\
\hline LVH on ECG & 1.71 & $0.93-3.15$ & 0.09 \\
\hline
\end{tabular}




\begin{tabular}{|c|c|c|c|}
\hline & OR & $95 \% \mathrm{Cl}$ & $p$ value \\
\hline \multicolumn{4}{|c|}{ Composite outcome (death or admission at 1 year) } \\
\hline Troponin & 2.06 & $1.25-3.39$ & 0.005 \\
\hline History of CAD & 2.10 & $1.26-3.52$ & 0.005 \\
\hline eGFR* & 0.99 & $0.98-1.0$ & 0.004 \\
\hline DNR & 2.36 & $1.11-5.04$ & 0.03 \\
\hline LVH on ECG & 2.46 & $1.29-4.69$ & 0.006 \\
\hline \multicolumn{4}{|l|}{ Death at 1 year } \\
\hline Troponin & 1.34 & $0.62-2.89$ & 0.5 \\
\hline History of CAD & 3.65 & $1.78-7.48$ & $<0.001$ \\
\hline eGFR* & 0.99 & $0.98-1.0$ & 0.2 \\
\hline DNR status & 2.66 & $1.09-6.54$ & 0.03 \\
\hline LVH on ECG & 2.15 & $0.93-4.98$ & 0.07 \\
\hline \multicolumn{4}{|c|}{ Readmission at 1 year } \\
\hline Troponin & 2.02 & $1.22-3.36$ & 0.006 \\
\hline History of CAD & 2.44 & $1.45-4.08$ & 0.001 \\
\hline eGFR* & 0.99 & $0.98-1.00$ & 0.005 \\
\hline DNR status & 1.76 & $0.83-3.71$ & 0.1 \\
\hline LVH on ECG & 2.54 & $1.33-4.86$ & 0.005 \\
\hline \multicolumn{4}{|l|}{ Revisit at 1 month } \\
\hline Troponin & 1.44 & $0.85-2.44$ & 0.2 \\
\hline History of CAD & 0.96 & $0.56-1.66$ & 1.0 \\
\hline eGFR* & 1.00 & $0.99-1.01$ & 0.9 \\
\hline DNR status & 1.38 & $0.65-2.95$ & 0.4 \\
\hline LVH on ECG & 1.69 & $0.91-3.15$ & 0.1 \\
\hline
\end{tabular}

